\title{
CYCLICAL SUBNORMAL SEPARATED A-GROUPS OF NILPOTENT LENGTH FOUR
}

U. M. MAKARFI

(Received 22 November. 20(0)5: Revision Accepled 3 February. 2006)

\section{ABSTRACT}

In this paper, it is shown that a cyclically subnormally separated A-group of nilpotent length four exists. An A-group is constructed, shown to be of nilpotent length four and to be in the class of CSn groups.

KEYWORDS: CSn, A-groups of riipotent length four.

\section{INTRODUCTION}

The class of finite groups that we call CSn-aroups was introduced in (Makarfi,1991), while in (Makarfi, 2005b) the general question of A-groups in CSn was discussed by examining the monolithic A-groups. It was in that paper the issue of the bounds of the nilpotent length of CSn A-groups was raised. The purpose of this paper is to show that there are A-groups of nilpotent length four in CSn.

We know that the abelian groups provide the simplest examples of CSn A-groups of nilpotent length one. The metabelian groups were shown to be CSn groups, Lemma (3.5) of (Makarfi 1991), so they provide examples of CSn groups of nilpotent length two. A detailed description of A-groups in CSn of nilpotent length three was given in (" lakarfi, 1997a). So it is natural to look at those of nilpotent length four.

In section two we bring together those results that will help us through the construction and enable us to give the necessary proofs. The actual construction is given in section three. The schematic diagrams are aimed at helping the mind to visualize the type of the subgroups involved.

PRELIMINARIES

We start with the following result which has a serious consequence on the derived length of A-groups in CSn.

\section{Theorem 2.1}

Suppose that $G$ is an A-group in CSn then in $G / F$, every element of prime order is in $F_{2} / F$ where $F$ and $F_{2}$ are the Filing and the Second Fitting subgroups of $G$.

Proof: We note that the theorem says that if $x \in G$ and $x F$ has prime order in $G / F$ then $x \in F_{2}$. So to prove the theorem It is enough to show that if for some prime $p_{1} x^{p} \in F$ then $x \in F_{2}$. Without loss of generality we may take $x^{\prime}$ to be a $p$ element. Now if $\bar{G}=\mathrm{G} / \mathrm{F}$ and $\bar{x}=\mathrm{xF}$ then $\langle\bar{x}\rangle$ is cyclic of prime order. Also $\langle\bar{x}\rangle$ acts on $\bar{F}_{2}=F_{2} / F$ nonirivially, for otherwise,

$$
\overline{\mathrm{x}} \varepsilon \mathrm{C}_{\overline{\mathrm{G}}}\left(\overline{\mathrm{F}}_{2}\right) \subseteq \overline{\mathrm{F}}_{2}
$$

and this contradicts the assumption that $\mathrm{x}$ is not. in $\mathrm{F}_{2}$. So for some prime $\mathrm{q}$ not equal to $\mathrm{p}, \bar{x}$ acts non-trivially on some Sylow q-subgroup of $\bar{F}_{2}$.

The following diagram (Fig. 1) gives an idea of the kind of subgroups, in G, that we shall be dealing with. 


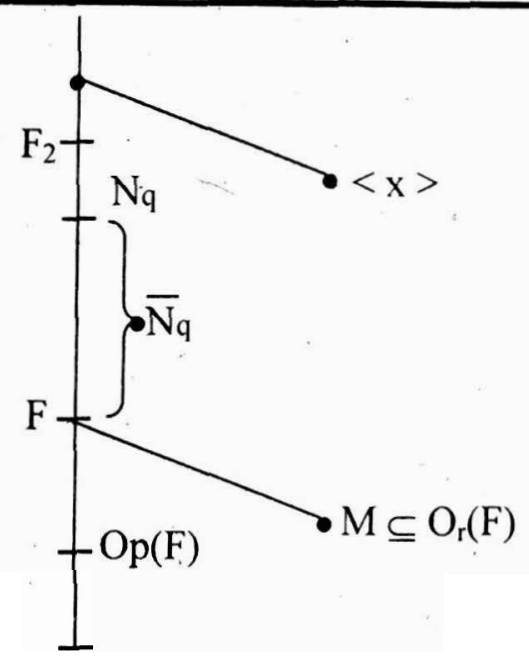

Fig. 1

Let $\overline{\mathrm{N}}_{\mathrm{q}}$ be a minimal q-subgroup of $\bar{F}_{2}$ such that

$$
\left[\bar{N}_{q}, \bar{x}\right]=\bar{N}_{q}
$$

Then $\bar{N}_{q}$ is elementary abelian subgroup of $\bar{F}_{2}$. Put $\mathrm{N}_{\mathrm{q}}<\mathrm{x}>=\mathrm{T}$. Then

$$
\mathrm{O}_{\mathrm{P}}(\mathrm{F}) \triangleleft T
$$

and

$$
\begin{aligned}
& x \in \mathrm{C}_{\mathrm{T}}\left(\mathrm{O}_{\mathrm{p}}(\mathrm{F})\right) \\
& {\left[\mathrm{N}_{\mathrm{q}}, \mathrm{X}\right] \subseteq \mathrm{C}_{\mathrm{T}}\left(\mathrm{O}_{\mathrm{p}}(\mathrm{F})\right)}
\end{aligned}
$$

Now

$$
\begin{aligned}
& N_{q}=F\left[N_{q}, x\right] \\
\therefore \quad & O_{p}(F) \subseteq Z\left(N_{q}\right) .
\end{aligned}
$$

We, next note that $x$ normalizes a Sylow $q$-subgroup, say

$\frac{O_{1}}{\sigma_{p}}(F)$ of $\mathrm{N}_{\mathrm{q}} / O_{p}(F)$ by co-prime action. Also $\mathrm{Q}_{1}$ is nilpotent, therefore

$$
Q_{1}=O_{p}(F) \times Q
$$

where $Q$ is an $O_{q}\left(Q_{1}\right)$. Because $Q$ is characteristic, in $Q_{1}$, we have that $Q$ is $x$ invariant. The next thing to observe is that $Q$ acts on $O_{p}^{\prime}(F)$ non-trivially, otherwise $Q$ will centralize $F$, which is not the case. So for some prime $r \neq p, q$ we can choose $M \subseteq O_{r}(F)$ a Sylow r-subgroup of $F, ' M$ minimal subject to being $Q<x>-$ invariant and

$$
[M, Q]=M
$$

So that $M$ is also elementary abelian. We then form the group

$$
L=M Q<x>
$$


and by lemma (2.4) below

$$
C_{M}(x) \neq-1
$$

We next let

$$
<x>^{\cdot G}=\mathrm{S}
$$

where $<x>G_{\text {is }}$ the subnormal closure of $x$ in $G$, then

$$
\bar{S}=<\bar{x}>{ }^{\cdots} \bar{G}
$$

and therefore $\bar{S} \supseteq \overline{\mathrm{N}}_{\mathrm{q}}$. Here we are using the fact that

$$
\begin{aligned}
\bar{N}_{q}=\left[\bar{N}_{q}, \bar{x}\right] \subseteq & {\left[\bar{N}_{4},<\bar{x}>^{\bar{c}}\right] } \\
& =\left[\bar{N}_{4}, \bar{S}\right] \\
& =\left[\bar{N}_{4}, \bar{S}, \bar{S}\right] \text { by co-prime action } \\
& \subseteq \bar{S}
\end{aligned}
$$

Therefore $\mathrm{N}_{\mathrm{q}} \subseteq \mathrm{SF}$. But

$[\mathrm{M}, \mathrm{SF}]=[\mathrm{M}, \mathrm{S}]$

$$
M=\left[M, N_{q}\right] \subseteq[M, S]
$$

Also we have

$$
M \subseteq[M, S]=[M, S, S] \subseteq S
$$

Thus

$$
M \subseteq<x>\cdot G
$$

and this contradicts the assumption that $\mathrm{G}$ is in $\mathrm{CS}$ n.

The next result is rather remarkable for it asserts that for an A-group inesn, some restrictions on the exponents of the Sylow subgroups puts a bound, on the nilpotent length of the group.

\section{Theorem (2.2)}

Let $\mathrm{G}$ be an $\mathrm{A}$-group in $\mathrm{CSn}$ such that $\mathrm{G} / \mathrm{F}$ is generated by elements of order dividing $P$," for distinct primes $p_{i}$ and fixed integer $n$. Then

$$
\mathrm{G}^{(n+1)}=1
$$

Proof: By induction on $n$. If $n=1$ then $G / F$ is generated by elements of prime order and so by theorem (2.1) above $G=$ $F_{2}$ and hence metabelian. Therefore

$$
G^{(2)}=1
$$


For $n>1$ we again use theorem $(2.1)$ to see that $\cdot\left(j / r_{2}\right.$ is generated by elements of order $p_{1}^{n-1}$. So that if $\bar{G}=\bar{G} / F$ then $\bar{G} / F(\bar{G})$ is generated by elements of order $p_{1}^{\prime \prime-1}$. So by induction

$$
\bar{G}^{(n)}=1
$$

and hence

$$
\bar{G}^{(n+1)}=1
$$

The following results will be needed as we go along with the construction.

Lemma (2.3) (Lemma (3.5) of Makarfi, 1991)

If $G$ is a metabelian group then $G$ is in CSn

Lemma (2.4) (Theorem (2.5) of Makarfi, 2005a)

Suppose that $G=V_{3} G_{2}$ is a monolithic group with monolith $V_{3}$ which is an elementary abelian q-group, where $G_{2}=V_{2} G_{1}$ is also monolithic with monolith $V_{2}$ an elementary abelian p-group and $G_{1}=\langle x\rangle$ is cyclic of order $r$, such that $q, p$ and $r$ are distinct primes. Then

$$
\mathrm{C}_{\mathrm{V} 3}(\mathrm{x}) \neq \mathrm{O}
$$

i.e. $x$ has a nontrivial centraliser in $V_{3}$.

Theorem (2.5) (Theorem C of Makarfi, 2005a)

Suppose that $G=W \quad X H$ is a $n$ A-group where $W$ is a minimal normal p-subgroup of $G$ for some prime $p$, and $H$ is a metabelian $p^{\prime}$-subgroup acting faithfully on $W$. Then $G$ is a CSn group if and only if every element of $\mathrm{HiF}(\mathrm{H})$ acts fixed-point-freely on $W_{i}$ where $F(H)$ is the Fitting subgroup of $H$.

Theorem (2.6) (Theorem A of Makarfi, 2005b)

Let $H$ be a group and $V$ a p-group which is an irreducible $k H$-module, where $k=F_{p}$ is a splitting field for $H$ and $p$ is a prime. Suppose that $G=V 4$.is an A-group, then $G$ is in CSn if and only if the following two conditions are satisfied.

(i) $\quad H$ is a CSn group and there exists $L \triangleleft K \subseteq H$ with $K / L$ cyclic and a faithful and irreducible $k[K / L]$ module $\mathrm{U}$ such that $\mathrm{V}=\mathrm{U}^{\mathrm{H}}$.

(ii) $L$ and $K$ can be chosen such that $L$ is subnormal in $H$ and for all $p^{\prime}$-elements, $x$ in $H$, of prime power order $<\mathrm{x}>\bigcap \mathrm{K}=<\mathrm{x}>\bigcap \mathrm{L} \Leftrightarrow \mathrm{x} \varepsilon \mathrm{L}$

Theorem 2.7 (Makarfi, 1997b)

Let $G$ be an A-group of nilpotent length $n$ and let

$$
1=G^{(n)} \subset G^{(n-1)} \subset \ldots \subset G^{(1)} \subset G^{(0)}=G
$$


be the derived series of $G$ then

(i) $\mathrm{G}=\mathrm{A}_{n-1} \mathrm{~A}_{\mathrm{n}-2} \ldots \mathrm{A}_{0}$; where the $A_{i}^{*}$ are abelian.

(ii) $\quad G^{(n-i)}=A_{n-1} A_{n-2} . . A_{n-i}$ for each $i \in\{1,2, \ldots, n\}$

(iii) $\quad \mathrm{F}=\mathrm{A}_{\mathrm{n}-1} \times F \cap A_{n-2} x \ldots x F \cap A_{0}$; where $\mathrm{F}$ is the Fitting subgroup of $\mathrm{G}$.

(iv) $\quad Z\left(G^{(n-i)}\right)=F \cap A_{n-i}$ for each i $\in\{1,2, \ldots, n\}$

where $Z\left(G^{(r)}\right)$ is the centre of $G^{(r)}$.

\section{CONSTRUCTION AND PROOF} a CSn-group.

In this section we shall construct an A-group of nilpotent length four and show that the group so constructed is

We start by introducing a diagram (Fig. 2) to give a schematic idea of the subgroups of prime power orders involved and the various other sub-groups we shall be interested as we go through the construction. The vertical columns give the subgroups of prime power orders, while the horizontal columns give the other sub-groups we get involved with.

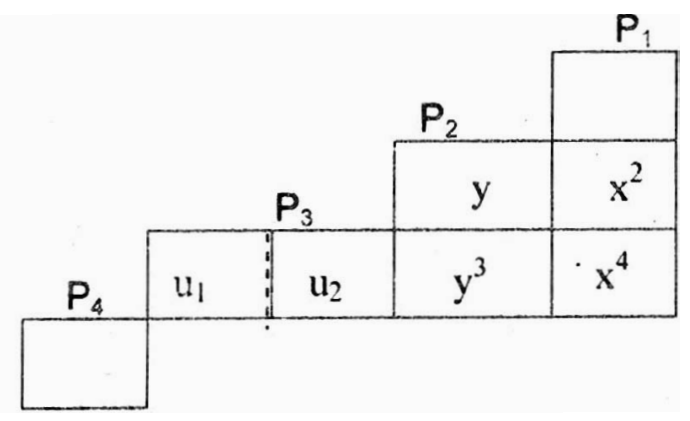

Fig. 2

Now let $P_{1}=C_{8}=\langle x\rangle$ be a cyclic 2-group of order eight and let $P_{2}=C_{3}=\langle y\rangle$ be a cyclic 3-group of order -ne. Let $P_{1}$ act on $P_{2}$ by conjugation as follows: $y^{x}=y^{-1}$ and form the group

$$
A=P_{2} \searrow P_{1}
$$

So that

$$
A=\left\langle x, y \mid x^{8}=y^{9}=1, y^{x}=y^{-1}\right\rangle
$$

Next, let $M=\left\langle y, x^{2}\right\rangle$, then $M$ is a normal abelian subgroup of $A . M$ has a 1-dimensional module $U=k, u$, where $k_{1}=F_{7}$, via the following action

$$
y: u \longmapsto 2 u \text { and } x^{2}: u \longmapsto-u
$$

$$
P_{3}=U^{A}=\left\langle u_{1}, u_{2} \mid u_{1}^{7}=u_{2}^{7}=\left[u_{1}, u_{2}\right]=1\right\rangle
$$

where $u_{1}=u \otimes, 1$ and $u_{2}=u \otimes x$. We are here using $\{1, x\}$ as a transversal to $M$ in $A$. We then have the action of $y$ and $x$ on $P_{3}$ as follows: 
$u_{1} y=2 u_{1}, u_{2} v=(u \otimes x) y=u \otimes y^{x-1} x=u \otimes y^{-1} x=4 u_{2}$

i.e. $u_{1} y=2 u_{1}, u_{2} y=4 u_{2}, u_{1} x=u_{2}$ and $u_{2} x=-u_{1}$. So that the action can be represented as

$$
\left.\begin{array}{l}
y \rightarrow\left(\begin{array}{ll}
2 & 0 \\
0 & 4
\end{array}\right) \\
x \rightarrow\left(\begin{array}{rr}
0 & -1 \\
1 & 0
\end{array}\right)
\end{array}\right\} \operatorname{Mod} 7
$$

\section{Mod 7}

We then form the group

$\mathrm{H}=\mathrm{P}_{3} \rtimes \mathrm{A}=<\mathrm{x}, \mathrm{y}, \mathrm{u}_{1}, u_{2} \mid x^{8}=y^{9}=u_{1}^{7}=u_{2}^{7}=\left[u_{1}, u_{2}\right]=1, u_{1}^{x}=u_{2}, u_{2}^{x}=u_{1}^{-1}, u_{1}^{y}=u_{1}^{2}, u_{2}^{y}=u_{2}^{4}>$

Now let

$$
K=\left\langle u_{1}, u_{2}, y^{3}, x^{4}\right\rangle
$$

then $K$ is a normal abelian sub-group of $H$. In fact $K$ is the Fitting subgroup of $H$. Supposed that $k_{2}=F_{43}$ then $W=k_{2} W$ can be turned into a 1-dimensional K-module through the following action

$$
w u_{1}=21 w, w u_{2}=w ; w y^{3}=6 w \text { and } w x^{4}=-w
$$

We note that $F_{43}^{x}=<3>$ is the multiplicative group of the non zero elements of $F_{43}$ so that 21 has order seven and 6 has order three in $F_{43}$

The next step is to let $P_{4}=W^{H}$ To describe $P_{4}$ we note that $K$ has index twelve in $H$, so we may take a transversal

$$
\because T=\left\{1, y, y^{2}, x, y x, y^{2} x, x^{2}, y x^{2}, y^{2} x^{2}, x^{3}, y x^{3}, y^{2} x^{3}\right\}
$$

for $\mathrm{K}$ in $\mathrm{H} . \mathrm{W}^{\mathrm{H}}$ can then be described as

$$
W^{H}=\left\langle w_{i j}=w \otimes y^{i} x^{j}\right|\left(w_{i j}\right)^{43}=\left[w_{i j}, w_{r s}\right]=1 ; 0 \leq i, r \leq 2 ; 0 \leq j, s \leq 3>
$$

Finally, we form

$$
G=P_{4} \backslash H
$$

This is our group. What remains is to show that it is an A-group of nilpotent length four and that it is a CSn group.

By construction

$$
G=P_{4} P_{3} P_{2} P_{1}
$$

and it is clear that it is an A-group since the $P_{r}^{\prime}$; $1 \leq r \leq 4$ are Sylow subgroups, and are abelian. Also

$$
G^{(1)}=P_{4} P_{3} P_{2} ; G^{(2)}=P_{4} P_{3} ; G^{(3)}=P_{4} ; G^{(4)}=1
$$

hence $G$ has derived length (and therefore nilpotent length) four. 
To sbow that $G$ is in $C S n$ we first note that $P_{1}$ being abelian is in CSn. Then $A=P_{2} \searrow \quad P_{1}$ is metabelian and cherefore in CSn by Lemma (2.3) above. Next

$$
\mathrm{H}=\mathrm{P}_{3} \searrow \mathrm{A}
$$

nas nilpotent length three so by theorem (2.5) $\mathrm{H}$ is in CSn if and only if every element of AIM act fixed point freely on $P_{3}$. Note that $M$ is the Fitting subgroup of $H$. Also in the course of proving theorem (2.5) it was shown that it is enough to consider only those elements of AIM of prime power order. But every such element is conjugate to $x$ and $x$ is fixed point free on $P_{3}$. The next step is to note that

$$
G=P_{4} \searrow H
$$

satisfies the hypotheses of theorem (2.6). Since condition (i) is satisfied by

$H, V=P_{4}, U=W, L=\left\langle u_{2}\right\rangle$ and $K=\left\langle u_{1}, u_{2}, y^{3}, x^{4}\right\rangle$

Also

$$
\mathrm{L} \triangleleft \mathrm{K} \triangleleft \mathrm{H}
$$

and

$$
L \cap \mathrm{K}=<\mathrm{u}_{2}>\subset P_{3}
$$

Now suppose that $\mathrm{h}$ is a non trivial element of $\mathrm{H}$ of prime power order such that

$$
<h>\bigcap \mathrm{K}=<\mathrm{h}>\bigcap \mathrm{L}
$$

We know that $\mathrm{K}$ contains all the elements of $\mathrm{H}$ of prime order; since

$$
<\Omega_{1}\left(P_{3}\right), \Omega_{1}\left(P_{2}\right), \Omega_{1}\left(P_{1}\right)>
$$

is contained in $\mathrm{K}$ and each

$$
\Omega_{1}\left(P_{r}\right) ; 1 \leq r \leq 3
$$

is normal in $\mathrm{H}$. So $<h>\bigcap K \neq 1$ and $\mathrm{h}$ is a $\mathrm{p}_{3}$-element. Now $\mathrm{P}_{3}$ is elementary abelian and $\mathrm{L}$ is $\dot{c} y c l i c$ of order $\mathrm{p}_{3}$. So $h$ must be in $L$ and hence $G$ also satisfies condition (ii) of the theorem. Thus $G$ must be in CSn.

\section{CONCLUSION}

We have constructed an A-group of nilpotent length four and shown that it is in CSn

\section{ACKNOWLEDGEMENT}

The author wishes to thank the International Centre for Theoretical Physics, Trieste for giving him the facilities to prepare this paper.

\section{REFERENCES}

Makarfi, U. M., 1991. A class of Subgroups satisfying certain subnormal conditions. Afrika Mathamatika, Series 2(3): $65-82$. 
Makarfi, U. M., 2005a. Cyclical Subnormal Separation in A-groups. To appear in Global J. of Mathematical Sciences, Vol. 5 .

Makarfl, U. M., 2005b. Monolithic A-group in CSn. To appear in Global Journal of Mathematical Sciences, Volume 5.

Makarfi, U. M., 1997a. A characterization of Cyclic Subnormal Separated A-group of nilpotent length three. Bull. Austral, Math. Soc. 56: $243-251$.

Makarfi, U. M., 1997b. A theorem on A-groups: The Mathematics Education, India, vol: xxxi, No.3, pp. $138-143$. 